\title{
Quiasmo, carne y naturaleza. Merleau-Ponty y el problema de la medialidad
}

\author{
Nicolás Fagioli \\ Universidad de Buenos Aires/Universidad Nacional de las Artes, Argentina \\ nicofagioli@gmail.com \\ https://orcid.org/0000-0002-7263-9765
}

Resumen: La noción de carne, perteneciente a la última etapa del pensamiento de Merleau-Ponty, ocupa un lugar determinante en las investigaciones especializadas sobre el autor. Sin embargo, en escasas publicaciones se destaca la inmensa influencia de esta última en el pensamiento actual, especialmente en las corrientes posthumanistas y materialistas de la contemporaneidad filosófica. Nos proponemos, en las páginas que siguen, analizar dicha categoría desde el punto de vista de una filosofia de la relación, interpretándola desde la figura de la medialidad o emparentándola a una noción de medio. Para esto, en la primera parte, se dilucidará la noción merleau-pontiana de medio en Fenomenología de la percepción y la postulación de la experiencia como punto de partida. En la segunda, se explicitarán las múltiples dimensiones en las cuales se despliega la noción de carne en la filosofia tardía del autor. Una vez abordados los aspectos epistemológico, fenomenológico y ontológico de la ontología de la carne se analizará su potencial filosófico proyectando los tratamientos a la problemática de la naturaleza, cuestión que consideramos de vital importancia para las reflexiones filosóficas actuales.

Palabras clave: carne; quiasmo; medialidad; entrelazo; naturaleza

\begin{abstract}
Chiasm, Flesh and Nature. Merleau-Ponty and the Problem of Mediality". The notion of flesh, belonging to the last stage of Merleau-Ponty's thought, occupies a decisive place in specialized research on the author. However, few publications highlight the immense influence of the latter in current thought, especially in the post-humanist and materialist currents of contemporary philosophy. We propose, in the pages that follow, to analyze this category from the point of view of a philosophy of relationship, interpreting it from the figure of mediality or relating them to a notion of medium. For this, in the first part, we will elucidate the notion of medium used in Phenomenology of Perception and the postulation of experience as a starting point. In the second part, we will specify the multiple dimensions in which the notion of flesh unfolds in the late philosophy of the author. Once the epistemological, phenomenological and ontological aspects of the ontology of the flesh have been addressed, its philosophical potential will be analyzed, projecting the treatments for the problems of nature, an issue that we consider of vital importance for current philosophical reflections.
\end{abstract}

Keywords: flesh; chiasm; mediality; intertwining; nature 


\section{Introducción}

El presente escrito se enfocará en el pensamiento tardío de MerleauPonty, especialmente en los planteos desarrollados en su obra póstuma "El entrelazo - el quiasmo", capítulo final de Lo visible y lo invisible. El objetivo principal de nuestro desarrollo será postular a este "último" Merleau-Ponty dentro de lo que podriamos denominar una filosofía de la medialidad o de la relación ${ }^{1}$, en tanto enfoca el objeto de su investigación en el entrelazamiento entre los componentes de determinado binomio, en este caso, cuerpo-mundo, más que decantar por uno de ellos. Es decir, se le da espesor ontológico al vínculo en sí mismo considerándolo como un objeto legítimo de reflexión. Esta concepción funciona, por un lado, como una alternativa a la histórica dicotomía filosófica entre idealismo y realismo, anhelo presente en muchos pensadores de los siglos XX y XXI que intentan escapar de los dualismos modernos. Por tal razón, como segundo objetivo, creemos que concebir los planteos merleaupontianos desde una filosofia de este tipo ilumina numerosas problemáticas actuales, específicamente aquellas relacionadas a las discusiones en torno al concepto de naturaleza.

Algunos comentadores, como Hamrick y Van der Veken con especial énfasis, postulan que asistimos en esta etapa a una suerte de giro ontológico de la filosofia del francés. Este giro se manifestaría en el desplazamiento desde una teoría de la sensación, centrada en el cuerpo, hacia una teoría de lo sensible en si, cuyo centro sigue siendo el cuerpo, pero enfocado desde un nuevo lugar y trascendiendo las fronteras fenomenológicas o gnoseológicas. Si bien no nos involucraremos en este debate, nos limitaremos a decir que acordamos con Esteban García en que el matiz ontológico no es exclusivo de la última etapa de la obra merleau-pontiana ${ }^{2}$. En efecto, el cuerpo funciona como eje tanto ontológico como fenomenológico en la totalidad de la obra del autor y resulta un modelo para pensar tanto la experiencia corporal de modo integral, como al ser en general. De hecho, como veremos, es posible rastrear una noción de "medio", fundamental para nuestra investigación en la Fenomenología de la percepción.

\footnotetext{
1 Si bien pueden establecerse algunos matices entre estas dos acepciones, en el marco de nuestra investigación, pueden considerare como sinónimos.

2 Cf. García, E., Maurice Merleau-Ponty. Filosofia, corporalidad y percepción, Buenos Aires: Rhesis, 2012.
} 
Por lo tanto, será necesario para nuestra investigación remitirnos también a esta obra, además de la que nos ocupa principalmente. Si bien en Lo visible y lo invisible se explicita de un modo más evidente, "desde la Fenomenología la preocupación ontológica del filósofo se hace evidente no sólo en su apuesta explícita de definir 'un tercer tipo de ser' sino en su tematización del mundo y particularmente del "mundo natural"' 3 . Este tercer tipo de ser y ese mundo natural son los problemas que nos ocupan.

El trabajo está organizado en tres secciones. Se analizarán, en primer término algunos tratamientos de la noción de medio en Fenomenología de la percepción, y la postura epistemológica que una filosofia de la relación obliga a adoptar. En la segunda parte se explicitarán las dimensiones de la noción de carne y sus notas distintivas. En la tercera, se indagará en el modo en que estos planteos iluminan uno de los problemas ontológicos y éticos más relevantes de la actualidad filosófica: la cuestión de la naturaleza.

\section{Aclaraciones preliminares: Fundamentos de una filosofía de la medialidad}

En términos generales, concebiremos como una filosofia de la medialidad o de la relación a la investigación que pondera el vínculo como tal antes que los componentes de determinado sistema o binomio. Una problemática paradigmática dentro de este enfoque es la relación entre el individuo y su entorno. Dicha dualidad ha adquirido, históricamente, diferentes avatares (sujeto/ objeto, cuerpo/mundo, mente/materia, naturaleza/cultura) y las diferentes tendencias filosóficas siempre han decantado, salvo contadas excepciones, por uno u otro de los polos en tensión. Contra esto, la investigación en torno a una nueva noción de medio o la concepción de un tercer reino posibilita un abordaje diferente del dualismo filosófico heredado del pensamiento moderno, ya sea para desestimar la oposición tajante entre dos términos, evidenciar su codependencia, o explicitar fuerzas específicas encubiertas por la densidad conceptual de sus polos.

Una filosofia de la medialidad intentará enfocarse en este espacio intermedio con el fin de postularlo como un objeto legítimo de reflexión filosófica. Si bien será la filosofia del siglo XX la que le brinde un espacio significativo a estas tendencias no-substancialistas, la búsqueda de este lugar medial puede ser rastreada desde los primeros esbozos del pensamiento occidental. Podría decirse que la búsqueda de una idea de lo medial habita, más o menos subrep-

3 Garcia, E., Maurice Merleau-Ponty. Filosofia, corporalidad y percepción, p. 14. 
ticiamente, los grandes tópicos de la filosofia. De algún modo, es un problema que recorre toda la historia del pensamiento y, en mayor o menor medida, toda reflexión filosófica aborda, más no sea tangencialmente, esta cuestión. Quizás sean los filósofos injustamente llamados presocráticos los que más se conecten con esta inspiración, pero a partir de la inconmensurable figura de Platón, salvo contadas excepciones, si la reflexión filosófica aborda una figura de lo medial, lo hace como un objeto secundario y complementario.

Una filosofia tal debe escapar tanto del dualismo como del monismo: la relación no debe reducirse al análisis de los elementos que la componen, así como tampoco debe pretender unificarlos o sintetizarlos en una unidad trascendente. En palabras de Mario Teodoro Ramírez: "no hay contradicción o exclusión entre los componentes de la dualidad. Ellos son lo mismo sólo que esta mismidad es algo"4. Podría definirse también como una ontología que toma a la relación en sí misma como constitutiva de sus componentes, los cuales no están previamente dados ni son autosuficientes, sino que existen gracias a dicha relación.

Los componentes conceptuales no desaparecen ni deben ser desechados, sino enfocados desde una nueva perspectiva, observando el modo en que se afirman en la contraposición del binomio. La intención no es disolver el dualismo en pos de una unidad ingenua, ni decantar por uno de los polos sino mantenerse en un espacio medial con un espesor ontológico propio dentro del cual se difuminan sutilmente las fronteras entre los elementos, aunque no se eliminan del todo. Una filosofia que rehúye de la confrontación binaria y corre el foco de las sustancias a las correspondencias, de los polos a los intersticios, de lo estático al movimiento y los devenires.

En rigor, de diferentes modos, la mayor parte de la filosofia del siglo XX ha intentado escapar del dualismo moderno. El escape del antropocentrismo filosófico sólo puede venir de la mano de una verdadera filosofia de la relación que se enfrente a las tendencias subjetivistas y sustancialistas que, con contadas excepciones, dominaron la práctica filosófica desde las meditaciones cartesianas y la figura determinante de Kant.

En este sentido, Gilbert Simondon -quien, es preciso destacar, dedica su tesis doctoral a la memoria de Merleau-Ponty- sea quizás el nombre más

\footnotetext{
4 Ramírez, M. T., La filosofía del quiasmo. Introducción al pensamiento de Maurice Merleau Ponty, México D.F.: Fondo de Cultura Económica, 2013, p. 47.
} 
resonante y el que efectivamente ha utilizado esa expresión de manera explícita ${ }^{5}$. Sin embargo, otros pensadores del siglo pasado como Walter Benjamin pueden ser leídos también en esta clave, específicamente sus reflexiones en torno a la noción de entre desarrollada en La tarea del traductor. Del mismo modo, algunos trabajos de Giorgio Agamben, como continuador de Benjamin, o de Gilles Deleuze, como discípulo directo de Simondon, pueden ser considerados dentro de las filas de lo medial. En la actualidad, numerosos autores y corrientes podrian ser consideradas también participantes de este tipo de filosofia, como por ejemplo: el realismo agencial de Karen Barad con su noción de intra-actions; Rosi Braidotti, en el marco de los estudios posthumanistas, con sus planteos sobre un continuum naturaleza-cultura; Emanuele Coccia, en La vida de las plantas con su categoría de melange (mixtura) derivada de una filosofia de lo medial desarrollada en toda su obra.

\section{La noción de medio y el ser salvaje}

\subsection{El pensamiento del entre}

Si bien nos centraremos en la última etapa de su producción, la búsqueda merleau-pontiana por lo medial se encuentra anticipada en la Fenomenología, asociada al dualismo cuerpo-mundo: "El cuerpo es el vehículo del ser-delmundo, y poseer un cuerpo es para un viviente conectar con un medio definido, confundirse con ciertos proyectos y comprometerse continuamente con ellos"6. Que el cuerpo esté conectado con un medio implica que aquel adquiere una potencia definida por el vínculo generado. Como expone Emanuel Alloa en La resistencia de lo sensible: "En tanto potencialidad en un medio, el cuerpo maduro no se deja reducir ni a una autonomía total de un sujeto puro ni a la heteronomía de un entorno"7. Esto ubica a la noción merleau-pontiana de medio en un pensamiento del entre, en donde "entre [parmi] significa no sólo 'en un medio' sino por y a través de éste". En otras palabras, no es que el cuerpo se adapta a un medio que es a priori condicionante de aquel, es el vínculo el que define tanto las potencialidades como las condiciones.

De hecho, es el mismo cuerpo el que proyecta un medio en el mismo fenómeno de la percepción. En el capítulo "El sentir", el autor explica: "No

\footnotetext{
5 Para un análisis de la filosofia de la relación en Simondon ver la obra de Combes, M., Simondon. Una filosofía de lo transindividual, Buenos Aires: Cactus, 2017.

6 Merleau-Ponty, M., Fenomenología de la percepción, Madrid: Planeta, 1993, p. 100.

7 Alloa, E., La resistencia de lo sensible, Buenos Aires: Nueva Visión, 2009, p. 39.

8 Ibid., p. 36.
} 
quitamos la síntesis al cuerpo objetivo más que para darla al cuerpo fenomenal, eso es al cuerpo en cuanto proyecta a su alrededor un cierto 'medio' en cuanto que sus 'partes' se conocen dinámicamente una a otra y que sus receptores se disponen de modo que posibiliten con su sinergia la percepción del objeto"9. Como afirma Barbaras en el prólogo a la obra de Alloa citada hace un momento, el cuerpo es, en realidad, "mediación: es el medio del medio, en el doble sentido de su centro y de su medio. No sorprende entonces que, en el último MerleauPonty, la mediación llegue a ocupar el lugar de lo mediatizado, que ese medio que es el cuerpo -a la vez corazón del mundo y vector de su aparecer-, y que en lo sucesivo será nombrado Carne, entregue el sentido de ser verdadero de ese primer medio que es el mundo" 10 .

La cuestión del entre, resulta además el modo en que el mismo autor se posiciona ante la reflexión filosófica. En la introducción de Fenomenología de la percepción asegura: "Nos es preciso reconocer lo indeterminado como un fenómeno positivo"11. Este es un modo de anticipar lo que años más tarde llamaría quiasmo y conformaría el corazón de su ontología. Esteban García, a quien recurrimos nuevamente, destaca la positiva consideración de Merleau-Ponty hacia la permanencia en cierta ambigüedad con respecto a algunos problemas filosóficos como, por ejemplo, el de la problemática categoria de hábito. Este caso resulta paradigmático dado que es un concepto que se dilucida de manera más efectiva cuando la reflexión se ubica entre la naturaleza y la cultura, entre el pasado y el presente, entre la voluntad y los automatismos ${ }^{12}$. Así, el hábito es una categoría sedimentada en esa interzona que articula dos polos en tensión.

En efecto, si nos detenemos en la distinción entre las nociones de ambigüedad y ambivalencia realizada por García en el mismo escrito, notaremos que la primera se define de manera similar al modo en que, según nuestro análisis, el medio se enfrenta a la dualidad. Debemos concebir la ambigüedad como "la ambivalencia a la cual se atreve uno a mirar de frente, y consiste en admitir que un mismo ser puede ser a la vez una cosa y su contraria, o incluso ninguna de las dos exactamente sino algo más 'entre' ellas"13. Más adelante, García se pregunta: “¿Acaso no consiste la entera filosofia de Merleau-Ponty en

\footnotetext{
9 Merleau-Ponty, M., Fenomenología de la percepción, p. 247.

${ }^{10}$ Alloa, E., La resistencia de lo sensible, p. 10.

${ }_{11}$ Merleau-Ponty, M., Fenomenología de la percepción, p. 28.

${ }^{12}$ Cf. García, E., "¿Qué es un hábito? Acerca de la posibilidad de una desambiguación de la noción merleau-pontiana", en: Ideas. Revista de Filosofia Moderna y Contemporánea, v. III, 6 (2017), p. 54.

13 Ibid., p. 67
} 
este mismo intento de fijar la mirada en fenómenos de transición, y de forjar nuevos conceptos para pensar en lo que no es 'ni... ni...', otorgando a la ambigüedad del 'entre' un estatuto positivo?"14. Si lo que pretende pensarse es lo que no es ni una cosa, ni la otra, será necesario considerar a ese entre, como un objeto de análisis específico.

\subsection{Una cuestión de fe}

De este modo, vemos que el problema de la medialidad como legítimo terreno de disputa filosófica se encuentra presente desde mucho antes de concebir una obra como Lo visible y lo invisible. Sin embargo, será en esta última en donde se desarrolle más profundamente y en la que se pone en práctica una estricta conceptualización de la cuestión: este carácter paradojal, lo indeterminado y la ambigüedad, se integran ahora en el concepto de carne, y la dualidad cuerpo-mundo se reposiciona. En palabras de Alloa, lo que ocurre en esta etapa es una "radicalización" de la noción de medio, por medio de la cual: “el cuerpo ya no es un 'medio' en un mediomundo; cuerpo y mundo proceden de un tejido común, tienen que ver con un 'medio formador"'15. Este "tejido común" debe entenderse como una dimensión ontológicamente anterior a la división entre sujeto y objeto, una vida pre-reflexiva que resignifica la diferencia entre reflexión e intuición, así como también el modo de entender la experiencia de los sentidos.

Como explican Hamrick y Van der Veken, los planteos tardíos merleaupontianos introducen una ontología no dualista sino "dual" o "doble" (twofold) para la cual "mentes y cuerpos, espíritu y naturaleza, vida y materia, deben ser concebidos como reintegrados a una unidad más fundamental en orden a entender (pensar) sus relaciones adecuadamente"16. Esta anterioridad preobjetiva y pre-subjetiva es caracterizada en la primera parte de Lo visible y lo invisible como un ser salvaje: "ese ámbito pre-espiritual sin el cual nada es pensable, ni siquiera el pensamiento"17.

El autor da comienzo a dicho escrito proponiendo que aquello que San Agustín asegura acerca del tiempo, a saber, que es perfectamente familiar para cada uno, pero ninguno de nosotros puede explicarlo a los demás, hay

\footnotetext{
14 Ibid., p. 67

15 Alloa, E., La resistencia de lo sensible, p. 90.

${ }^{16}$ Hamrick, W.S. y J. Van der Veken, Nature and Logos. A Whiteheadian Key to Merleau-Ponty's Fundamental Thought, Nueva York: New York University Press, 2011, p. 56. La traducción es mía.

17 Merleau-Ponty, M., Lo visible y lo invisible, 2010, Buenos Aires: Nueva visión, p. 17
} 
que decirlo del mundo ${ }^{18}$. La propuesta nos posiciona desde el comienzo en una paradoja. ¿Cómo debe dilucidarse ese ser salvaje? ¿Cuál es la certeza de que el pensamiento parte de ese ámbito pre-espiritual? El interrogante es sin duda un problema epistemológico. El autor no duda en recurrir a la noción de "fe" a la hora de definir nuestra actitud ante lo que se ofrece a la percepción. Lo visible y lo invisible comienza postulando a la "Fe perceptiva" como el punto de partida de toda reflexión.

Este es el puntapié inicial de la ontología dual de Merleau-Ponty a la que entendemos aquí en términos de medialidad. Ambos, experiencia y mundo poseen un mismo origen, y será el propio entrelazo el germen de un pensamiento de la medialidad, debemos partir de la "co-génesis de los seres humanos y el mundo"19. Lo que se pretende es que el discurso filosófico se instale en este lugar en que se da este encuentro primordial y aborde, como propone MerleauPonty, "experiencias que aún no hayan sido trabajadas, que nos ofrecen a la vez desordenadamente, el sujeto y el objeto"20.

Por lo tanto, para concebir la posibilidad de pensar por fuera de la oposición irreversible del dualismo, el punto de partida debe desandar los caminos heredados del pensamiento científico y filosófico moderno: o bien el subjetivismo extremo o bien un materialismo ingenuo. Tanto en su vertiente racionalista, empirista o crítica, el occidente moderno aborda el fenómeno de la percepción como un evento más del mundo. Estudia la experiencia como si pudiera observarla desde un posicionamiento exterior. Esto decanta, inevitablemente, en una filosofia de la subjetividad fundada en la omnipresencia de un sujeto constituyente que se apropia del mundo y lo configura a través de sus categorías.

Para Merleau-Ponty, en cambio, la sensibilidad es más bien un modo de dinamitar la omnipresencia del sujeto, es una puerta hacia la interrogación por los fundamentos. Este es el único modo de construir una ontología que recupere al mundo que los modernos relegaron a un lugar marginal. Como afirma Mario Teodoro Ramírez: "Por el camino de la existencia sensible, salimos de Yo y retomamos al Mundo"21.

18 Cf. ibid., p. 17.

${ }^{19}$ Hamrick, W.S. y J. Van der Veken, Nature and Logos. A Whiteheadian Key to Merleau-Ponty's Fundamental Thought, p. 62.

${ }^{20}$ Cf. Merleau-Ponty, M., Lo visible y lo invisible, p. 119.

${ }^{21}$ Véase Ramírez, M.T., La filosofia del quiasmo. Introducción al pensamiento de Maurice MerleauPonty, p. 101. 
La percepción es el único punto de partida, como lo fue también para Descartes, con la diferencia de que la meta no es fortalecer los muros de la subjetividad sino justamente disolver las fronteras. El encuentro primordial del cuerpo con el mundo es posicionado como el grado cero de toda reflexión. En efecto, todo pensamiento es en sí mismo percepción, en tanto que es imposible adoptar de hecho una posición exterior a ella que nos permita sobrevolarla.

\section{Medio, quiasmo, carne}

En las producciones tardías de Merleau-Ponty la explicitación de la noción de carne se concentra principalmente en el ya mencionado apartado "El entrelazo - el quiasmo". Para explicitar el modo en que dicho concepto se vincula con una filosofia de la medialidad, lo dilucidaremos a través de una serie de dimensiones las cuales, según creemos, iluminan diferentes aspectos de la carne como medio 22 .

La primera de ellas refiere a la carne entendida como quiasmo y visibilidad. Apunta al carácter paradójico de la relación entre cuerpo y mundo. En términos generales, podemos caracterizar el quiasmo como un cruce, un encuentro o un entrecruzamiento. En el uso común existen dos fuentes principales del término: una perteneciente a la óptica (chiasma) y la otra a la retórica (chiasmus) ${ }^{23}$. En el caso de quiasmo óptico, refiere al solapamiento de las imágenes brindadas por ambos ojos, que se encuentran en una sola imagen dando lugar a la visión en tres dimensiones. En el caso del quiasmo retórico, es una figura del discurso cuyos elementos se disponen en un orden AB-BA repitiendo la estructura sintáctica. Dicho de otra manera, intercambia sus elementos volviendo reversible el sentido, dando lugar a una significación cruzada.

En ambos casos se alude a una unidad dual en cuyas partes componentes conviven mismidad y diferencia. El quiasmo merleau-pontiano es introducido

\footnotetext{
${ }^{22}$ Varios comentadores especializados en esta etapa del pensamiento merleau-pontiano han desglosado analíticamente el concepto a partir de esta estrategia de explicitar subconceptos que ayuden a la dilucidación. Algunos de ellos son: Hass, L., Merleau-Ponty's Philosophy, Indiana: Indiana University Press, 2008, pp. 137-144; Toadvine, T., Merleau-Ponty's Philosophy of Nature, Evanston- Illinois: Northwestern University Press, 2009, pp. 107-130; Hamrick, W.S. y J. Van der Veken, Nature and Logos. A Whiteheadian Key to Merleau-Ponty's Fundamental Thought, Nueva York: New York University Press, 2011, pp. 73-102; Carbone, M., The Flesh of Images. MerleauPonty Between Painting and Cinema, Nueva York: Nueva York University Press, 2015, pp. 1-7.

${ }^{23}$ Ambas significaciones remiten a la voz griega (chiasmos), en la cual se evidencia el arraigo material de su significado.
} 
a la cuestión de la percepción del siguiente modo: "Lo visible alrededor de nosotros parece descansar en sí mismo. Es como si nuestra visión se formara en su centro o como si hubiera entre él y nosotros una intimidad tan estrecha como la del mar y la playa. Y, sin embargo, no es posible que nos fundamos en él, ni que él pase a nosotros, porque entonces la visión se desvanecería en el momento mismo de hacerse, por desaparición del vidente o de lo visible"24. Ahora bien, ¿cómo es posible que el mundo percibido, las cosas, se presenten, por un lado, como excediendo su apariencia, no revelándosenos completamente, y por el otro, a la vez, accedemos a ellas de un modo acorde a su apariencia, es decir reducidas a la experiencia subjetiva de la percepción individual?

Desde una metafísica de la identidad, se podría concluir que esto sucede gracias a nuestro acceso limitado al mundo, derivando de ello que existe una realidad más allá de este acceso parcial, un ser completo que escaparía a nuestra percepción actual. Desde el pensamiento del quiasmo, esta dualidad es lo real mismo, lo que fundamenta el sentido de las cosas, y la posibilidad de su visibilidad. Cuando un determinado visible/tangible se encuentra rodeado por todo lo visible/tangible de su alrededor (cuando se da el quiasma óptico) ocurre lo que el autor denomina un narcisismo, en el cual el vidente es mirado a la vez por las cosas y su percepción es a la vez activa y pasiva. Merleau-Ponty condensa esta paradójica situación en el siguiente pasaje: “...el vidente, atrapado por lo que ve, no ve más que a sí mismo: hay entonces un narcisismo fundamental de toda visión; y, por la misma razón, la visión que él ejerce también la sufre por parte de las cosas. Como lo han expresado muchos pintores, yo me siento mirado por las cosas, mi actividad es idénticamente pasividad -lo cual es el sentido segundo y más profundo del narcisismo: no ver en el afuera, como ven los demás, el contorno del cuerpo que uno habita, sino sobre todo ser visto a través de él, existir en él, emigrar a él, ser seducido, captado, alienado por el fantasma, de manera que vidente y visible se remiten el uno al otro, y ya no se sabe quién ve y quién es visto" 25 . El vidente se encuentra "atrapado por lo que ve": el acto de mirar implica, a su vez, el estar siendo mirado. Este quiasmo entre actividad y pasividad, entre acción y pasión, es la esencia del narcisismo.

El carácter quiasmático de la relación entre cuerpo y mundo es el propio modo de ser de una sensibilidad general, la cual encarnamos. Por lo tanto, mirar y ser mirado son dos aspectos de un mismo movimiento. La cita concluye del siguiente modo: "Esa Visibilidad, esa generalidad de lo Sensible en sí, ese

${ }^{24}$ Merleau-Ponty, M., Lo visible y lo invisible, p. 119

${ }^{25}$ Ibid., p. 126.

ARETÉ Revista de Filosofia, v. XXXIII, 1, 2021 / e-ISSN 2223-3741 
anonimato innato de Mí-mismo, es lo que llamábamos carne hace un momento, y que, bien sabemos, carece en la filosofia tradicional de un término que lo designe"26. Acerca de esta complejidad del concepto de visibilidad, Carbone explica que a través de él, Merleau-Ponty, "no designa simplemente el ensamble de las cosas visibles. De hecho, para él, el término también incluye las líneas de fuerza y las dimensiones sugeridas por las cosas visibles como su propio horizonte interior o exterior" 27 . Esto es, dentro de la ontología de la carne, el quiasmo no es simplemente una superposición de imágenes, sino un estrecho de horizontes que denotan una superficie profunda. La Visibilidad, entendida a partir del quiasmo, incluye a lo potencialmente visible, a lo invisible, haciendo de la carne un ser de latencia. Un ser de verticalidad, cuya superficie visible (quale), es una de las infinitas capas de la carne del mundo.

La segunda de las dimensiones deriva directamente del carácter quiasmático de la relación cuerpo-mundo y refiere a la imposibilidad de que este carácter reversible, en donde no se sabe quién mira y quién es mirado, se consuma completamente. En otros términos, a la carne como "divergencia" (écart). Visible-invisible, sentido-sintiente, cuerpo-mundo, son mutuamente constitutivos y, a la vez, es su misma diferencia la que los define. También entendida como desvío o desviación, écart alude a una brecha, a una frontera difusa. Es, en palabras de Lawrence Hass, una "apertura diferencial del espacio en el corazón de la experiencia”28, un espacio abierto entre el cuerpo y el mundo, entre lo visible y lo invisible.

Alloa, analizando la noción a la luz del principio diacrítico saussureano, puntualiza en el hecho de que Merleau-Ponty juega con "el doble sentido de écarter tanto excluyendo y espacializando, produciendo una brecha (écart). De la misma forma en que los signos sólo reciben sus especificaciones desde el exterior, las marcas diacríticas son solamente operativas cuando se insertan dentro del campo de los signos"29. De este modo, la percepción depende de un campo de relaciones diferenciales. Al respecto, Alloa concluye: "La percepción en sí misma es un 'sistema diacrítico, relativo, oposicional' y los campos sensoriales representan ellos mismos 'sistemas diacriticos'... Lo que Merleau-Ponty entonces acredita a Saussure es el cambio de una noción de significado como

\footnotetext{
26 Ibid.

${ }^{27}$ Véase Carbone, M., The Flesh of Images. Merleau-Ponty Between Painting and Cinema, p. 1. La traducción es mía.

28 Hass, L. Merleau-Ponty's Philosophy, p. 137.

29 Alloa, E., "The Diacritical Nature of Meaning: Merleau-Ponty with Saussure", en: Chiasmi International, 15 (2013), p. 165.
} 
adjuntado a sustancias idénticas a significados como un proceso diferencial el cual concede a la negatividad toda su debida importancia" ${ }^{30}$. Ya sea como desvío o divergencia, el écart hace del quiasmo una unidad en la diferencia o una diferencia sin contradicción. En palabras de Mauro Carbone, la carne es "la textura unitaria en la cual cada cosa y cada cuerpo se manifiesta solamente como una diferencia con los otros cuerpos y las otras cosas" 31 .

A la luz del écart, la reversibilidad no es otra cosa que el vínculo que esa diferencia sin contradicción permite. Entender esta dialéctica entre écart y reversibilidad implica introducirse en el aspecto táctil del problema de la percepción. Basándose en los planteos de Husserl en Ideas II, Merleau-Ponty ilustra esta cuestión a través del muy transitado ejemplo de las manos: si con mi mano derecha toco mi mano izquierda, podría decir que la primera es tocante y la segunda tocada, sin embargo la primera, la que toca, también resulta receptora de sensaciones locales cuya fuente es la superficie de la segunda, la tocada. Lo que encontramos aquí es, como dice Barbaras, la "casi identidad" entre lo tocante y lo tocado: "cada mano es sucesivamente, o más bien casisimultáneamente, tocada y tocante"32.

Que la reflexividad no se consuma completamente resulta fundamental, ya que es un modo muy certero de pensar la idea de unidad en la diferencia antes mencionada. Si esta simultaneidad fuera completa, no existiría la percepción: es necesario que haya un recorrido en el espesor que divide lo tocante y lo tocado a través del cual se intercambian las funciones. La sensación es posible gracias a que el cuerpo es sintiente a la vez que sentido: sólo a condición de ser percibido, la sensibilidad puede encarnarse en un cuerpo. Si se consumara la reversibilidad, el quiasmo desaparece y se decanta o bien en una pura subjetividad o bien en una pura objetividad. Cuerpo y mundo mantienen una relación quiasmática, en tanto están solapados, mutuamente superpuestos, aunque manteniendo cada uno su ser.

A esta mutua incorporación entre lo percibido y lo percipiente es a lo que Merleau-Ponty grafica como una palpación con la mirada. El quiasmo trastoca la relación clásica entre la visión y el tacto, este último se convierte en la facultad constituyente del cuerpo vivido y la más determinante en nuestro vínculo con las cosas. Concordamos aquí con el análisis de Françoise Dastur, quien destaca

\footnotetext{
30 Ibid., p. 168.

31 Carbone, M., The Flesh of Images. Merleau-Ponty Between Painting and Cinema, p. 7.

32 Barbaras, R., The Being of the Phenomenon. Merleau-Ponty's Ontology, Indiana: Indiana University Press, 2004, p. 153. La traducción es mia.
} 
el privilegio ontológico que da el fenomenólogo francés al tacto por encima de la visión: "la reversibilidad entre lo interno y lo externo es estrictamente encontrada solamente en el tacto, el cual es de este modo el origen de su diferencia, la diferencia en la base a la cual la visión en sí misma puede ser establecida. Al tacto, por lo tanto, le es dado un sorprendente privilegio ontológico"33. Es en base a este privilegio que el autor concibe a lo visible como "tallado en lo tangible" y a este último como "prometido de alguna manera a la visibilidad" 34 . Lo tangible es una promesa para la vista y delimita sus caminos posibles.

Es por esto que "hay superposición y atravesamiento de territorio, no sólo entre lo tocado y quien toca, sino también entre lo tangible y lo visible" 35 . La visión colma y satura el espacio abierto por el tacto, por el movimiento, por la relación del cuerpo con el mundo. Este espacio abierto por el tacto no es una construcción o un mero ordenamiento, sino más bien un contrato. Es un trabajo en cooperación tanto entre los sentidos como entre el cuerpo y el mundo. Hay entre ellos una armonía, una pregnancia en la que, sin embargo, se conserva la tensión, un aire de familia que hace que el cuerpo sea una cosa entre las cosas sin dejar de ser un cuerpo que percibe. Somos capaces de sentir y de pensar el mundo que nos rodea gracias a que lo hacemos desde cierto lugar, desde un punto de vista perteneciente a lo sensible mismo. La perspectiva, el hecho de conocer el mundo a través de uno de sus aspectos no implica, entonces, un acceso limitado a él, sino que ese es el único modo en que el conocimiento puede darse. Si el acceso a las cosas no se diera a través de uno de sus aspectos, no sería necesario un cuerpo, ni sería posible el conocimiento.

Ahora bien, esta diferenciación constitutiva y fundamental es una resonancia de la diferencia que reside en todo lo existente. En esto reside la apuesta ontológica del filósofo francés, que Toadvine explica del siguiente modo: "La consecuencia es que el Ser no es primordialmente idéntico a sí mismo, sino un evento de no-diferencia originaria... La duplicidad de la cosa es la reverberación de la duplicidad del cuerpo y, ultimadamente del Ser"36. El problema de la duplicidad perceptual replica en la duplicidad del ser. Esto permite introducir en nuestro análisis la tercera dimensión de la carne, la cual refiere al parentesco ontológico que la sensibilidad instaura entre cuerpo y mundo: "El ser carnal,

\footnotetext{
${ }^{33}$ Evans, F. y L. Lawlor, Chiasms: Merleau-Ponty's Notion of Flesh, Nueva York: State University of New York Press, 2000, p. 40. La traducción es mía.

${ }^{34}$ Véase Merleau-Ponty, M., Lo visible y lo invisible, p. 122.

35 Ibid.

${ }^{36}$ Toadvine, T., Merleau-Ponty's Philosophy of Nature, p. 125.
} 
como ser de las profundidades, con numerosas hojas o numerosos aspectos, ser de latencia y presentación de una cierta ausencia, es un prototipo del Ser, del que nuestro cuerpo, sintiéndolo sensible, es una muy notable variante, pero cuya paradoja constitutiva ya está en todo lo visible"37. El carácter quiasmático constitutivo de la experiencia sensorial no es más que una variante de ese ser de latencia y potencia, que antes mencionábamos, y que es constitutivo de todo lo que puede ser percibido.

El parentesco se nos presenta como una diferencia infranqueable, pero a la vez como una intimidad: la percepción pareciera nacer desde adentro de lo visible, sin embargo, se mantiene la certeza de que una completa fusión entre esos dos órdenes es imposible. Renaud Barbaras reflexiona en torno a esta situación paradojal y concluye en que es, en realidad, la condición primera de la percepción: “...el espesor del cuerpo no impone una distancia entre la sensibilidad y el mundo. Por el contrario, la mera posibilidad de una experiencia sensible requiere este espesor. Es en virtud de este parentesco ontológico con el mundo, un parentesco conferido en ella por el cuerpo, que la conciencia puede abrirse al mundo. Una conciencia que no sería del mundo sería conciencia de nada, dado que fallaría en tener esta complicidad ontológica"38. Cuerpo y mundo forman parte de un mismo estilo de ser. Esto redefine, sin eliminarlas, las fronteras entre ellas, las convierte en un espesor. De allí la reversibilidad entre quien mira y quien es mirado. La experiencia y lo experienciado están hechos de la misma cosa, comparten las mismas dimensiones del ser.

La carne es, entonces, un parentesco constitutivo. Merleau-Ponty asegura que no es materia, ni espíritu, ni sustancia; el término que mejor la define es el de elemento. Invocando la voz presocrática del término, la carne en tanto elemento debe entenderse "en el sentido en que se lo usaba para hablar del agua, del aire, de la tierra y del fuego, es decir, en el sentido de una cosa general, a medio camino entre el individuo espacio-temporal y la idea, suerte de principio encarnado que importa un estilo de ser, en todas partes donde encuentra una parcela de él" ${ }^{39}$. No es un hecho "material" ni "espiritual" pero es, de hecho, condición de posibilidad de los hechos, lo que hace que el hecho sea tal. Hass explica: "Podemos pensarlo como un 'elemento de la experiencia', un elemento que está en juego donde sea que haya criaturas que perciban" ${ }^{40}$. Así como el aire,

37 Ibid., p. 124.

38 Barbaras, R., The Being of the Phenomenon. Merleau-Ponty's Ontology, p. 155.

39 Merleau-Ponty, M., Lo visible y lo invisible, p. 127.

40 Hass, L. Merleau-Ponty's Philosophy, p. 137. 
agua, tierra y fuego eran presupuestos en múltiples instanciaciones, la carne posee tantas instanciaciones y dimensiones como lo permite la experiencia. Este es el modo en que la carne redefine la relación entre cuerpo y mundo: 1o que era considerado el afuera, es convertido en una profundidad o un espesor que debe ser concebido más bien como una no-exterioridad constitutiva. La interioridad del sujeto es un pliegue de lo exterior y la supuesta privacidad de mi sensación es un pliegue de lo sensible del mundo.

\section{Medialidad y naturaleza}

Como mencionamos en la introducción del presente escrito, la principal consecuencia de nuestra lectura de la carne en tanto medialidad involucra al plano ontológico y ético, más específicamente en torno al problema de la naturaleza. Todo el recorrido, desde el temprano concepto de medio en la Fenomenología, pasando por la ontología de la carne, decanta en un concepto de naturaleza que la súbita muerte del autor no permitió desarrollar cabalmente. Creemos que, en tiempos de posthumanismos y nuevos materialismos, esta cuestión adquiere la mayor relevancia filosófica, y es en el ámbito en que una filosofia de la medialidad resulta de mayor utilidad.

La naturaleza puede ser otro modo de entender al concepto de carne, y podriamos considerarla de hecho como una cuarta dimensión de significado que armoniza con las tres anteriores. Existen varias motivaciones para realizar esta asociación. En primer lugar, entre 1959 y 1960, Merleau-Ponty dicta tres cursos en torno al concepto de naturaleza que se recopilaron póstumamente en la obra La Nature: Notes, cours du College de France. Los mismos fueron preparados en paralelo al proceso de escritura de Lo visible y lo invisible, el cual también quedó trunco y fue publicado luego de la muerte del autor. En La Nature se desarrolla una crítica a la concepción científica y filosófica del concepto de naturaleza a partir de una minuciosa reconstrucción histórica. Dicha noción es concebida en los mismos términos de parentesco ontológico que la carne; Merleau-Ponty conjetura en un lenguaje algo criptico: "La naturaleza en nosotros debe tener alguna relación con la Naturaleza afuera de nosotros, es más, la Naturaleza afuera de nosotros debe revelársenos por la Naturaleza que somos"41. La naturaleza no es externa al sintiente sino que también comparte un aire de familia con este, y su dilucidación debe partir de este vínculo profundo.

\footnotetext{
${ }^{41}$ Merleau-Ponty, M., La nature. Notes et cours du Collège de France, París: Éditions du Seuil, 2003, p. 206. La traducción es mía.
}

ARETÉ Revista de Filosofía, v. XXXIII, 1, 2021 / e-ISSN 2223-3741 
En segundo lugar, la propia explicitación del concepto de carne como elemento nos lleva a la asociación. ¿Existe acaso un gesto más potente por construir una filosofia de la naturaleza que invocar a la intuición presocrática? Si bien la naturaleza por la que interrogaban los presocráticos, entendida como arkhé, resulta bien distinta a la que se concebía en pleno Siglo XX, recurrir a la idea de elemento evoca aquella intuición. La búsqueda del fundamento se reconfigura aquí como un arkhé carnal. Si los antiguos buscaban el origen en un elemento de la naturaleza exterior, aquí el elemento surge del propio quiasmo entre el sujeto y el mundo. En la filosofia tardía de Merleau-Ponty, la naturaleza se nos presenta como anterior, como un tejido común que no es ni exterior ni interior, sino lo que funda esa dualidad. A partir de esto, entonces, tomamos como punto de partida que la naturaleza no puede pensarse por fuera de la experiencia.

Ahora bien, podemos preguntarnos: ¿cuál es la problemática de la naturaleza para la cual un pensamiento de la medialidad resultaría fructífero? Como dice Ted Toadvine en Merlau-Ponty's Philosophy of Nature, la pregunta acerca de la naturaleza se encuentra, en la actualidad, prácticamente olvidada. La sentencia, aunque con excesiva radicalidad, denota un problema real. Según el autor, en el transcurso del Siglo XX, especialmente en sus décadas finales, la cuestión de la naturaleza se ha inclinado paulatinamente hacia una ética ambiental, concentrada en los comportamientos y olvidando los profundos interrogantes filosóficos que subyacen a sus problemas.

De manera paradójica, a la proliferación de una conciencia ecológica le correspondió un olvido de la pregunta filosófica acerca de la naturaleza. De este modo, los objetos de pesquisa se reducen a cuestiones axiológicas indiferentes a problemas ontológicos de fondo, de los cuales el más importante apunta a dilucidar de qué hablamos cuando hablamos de naturaleza y cuál es nuestro vínculo con ella. Dicha indagación, a lo sumo, fue apropiada tímidamente por la filosofia de la ciencia pero con un enfoque enteramente distinto, preocupado más por querellas conceptuales que, aunque necesarias e indispensables, no poseían el mismo espíritu. A causa de esto, la pluralidad de la noción filosófica de naturaleza, de múltiples significados y conexiones, es reducida a un concepto técnico en el plano del análisis filosófico, y reemplazada por la del ambiente en el plano ético. En esta doble reducción se generan dos consecuencias: en primer lugar, se refuerza la visión occidental moderna de una naturaleza objetivable y disponible ante la cual el sintiente humano se posiciona, en el mejor de los casos, como mero espectador y, en el peor de ellos, como propietario y servidor. 
En segundo lugar, y derivado de esto, el ser humano es separado de la naturaleza generándose una división irreconciliable entre esta y la experiencia humana. Es por esto que, según Toadvine, desplazar el centro del problema desde la naturaleza al medio ambiente acarrea grandes contradicciones para un pensamiento que se pretende postantropocéntrico: "La noción de ambiente connota el mundo circundante, el escenario, e implicitamente esto significa el escenario para los seres humanos... El 'ambiente' como una reificación posicionada enfrente del sujeto humano, implica una división artificial entre la naturaleza y los humanos y nos alienta a ver la naturaleza como una colección de cosas más que en términos de relaciones mutuamente constitutivas" 42 .

Ahora bien, una vez establecido el problema, cabe preguntarnos el modo en que los planteos de Merleau Ponty resultarian fructíferos para este problema. Consideramos que a partir del acercamiento del concepto de naturaleza a la noción de carne es posible recuperar el lazo entre la naturaleza y la experiencia sensorial. Esta revinculación resulta fundamental dado que los problemas detallados por Toadvine evidencian una ruptura entre estas dos esferas. La naturaleza no puede ser considerada algo externo a la experiencia, sino que, por el contrario, el propio concepto de naturaleza debe extraerse de la experiencia. Para ello es necesaria la recuperación de la indagación ontológica de la cual se desprenda un nuevo concepto de naturaleza y, a partir de ella, alcanzar nuevos horizontes éticos. Se hace necesaria, entonces, una nueva ontología, una que, como explica Mauro Carbone, "pueda sustituir a la moderna, ésa que concibe a la Naturaleza como Objeto absoluto y al Sujeto como kosmotheorós, esto es, como su espectador igualmente absoluto"43. La naturaleza no puede ser un espectáculo para el viviente, pero tampoco puede considerársela como un mero medio condicionante. Una nueva filosofia de la naturaleza implica una nueva filosofia de la experiencia que armonice con las notas distintivas de la ontología de la carne.

\subsection{Hacia una nueva estructura de la experiencia}

Merleau-Ponty no es el primero en vislumbrar la cercana relación entre naturaleza y experiencia. Algunos años antes, Alfred North Whitehead, a quien nuestro autor dedica unas breves páginas en La Nature, había dilucidado un concepto de naturaleza similar. Merleau-Ponty destaca principalmente sus

\footnotetext{
42 Toadvine, T., Merleau-Ponty's Philosophy of Nature, p. 6.

${ }^{43}$ Carbone, M., "La naturaleza en Merleau-Ponty: variaciones sobre el tema", en: Devenires, v. I, 2 (2000), p. 167.
} 
tratamientos en torno a las ideas de espacio y tiempo y su crítica a la simple locación ${ }^{44}$, los cuales resultan fundamentales para sus propios desarrollos. Alli, el francés subraya: "La concepción tradicional de espacio y tiempo como contenedores, como 'aquello en lo cual la naturaleza está instalada' dispone una concepción de materia y substancia. La materia puede ser solamente una entidad substancial de la cual todos los fenómenos serán los atributos"45. Merleau-Ponty entiende, por un lado, que la búsqueda por un nuevo concepto de naturaleza conlleva el pasaje de lo que él denomina una 'ontología del objeto', o sea, una filosofia sustancialista, hacia una "ontología de lo existente"46.

Por otro lado, la naturaleza en tanto carne, en tanto entrelazo, es lo contrario a un contenedor. Espacio y tiempo deben ser abstracciones que se extraen de la naturaleza y no contenedores de esta. En Lo visible y lo invisible encontramos un razonamiento similar, pero esta vez en el marco de su teoría de la carne: "Tenemos que rechazar los prejuicios seculares que ponen el cuerpo en el mundo y el vidente en el cuerpo, o, inversamente, el mundo y el cuerpo en el vidente como en una caja. ¿Dónde poner el límite entre cuerpo y mundo, puesto que el mundo es carne?"47. La naturaleza-carne no puede concebir al espacio como un contenedor de los cuerpos, ni al cuerpo como un contenedor del vidente, readaptando la antigua concepción del cuerpo como prisión del alma. Espacio y tiempo deben comprenderse, entonces, a partir de la relación que se constituye entre nuestra percepción y aquello de lo que tenemos experiencia. Es el propio carácter del acontecimiento y su modo de ofrecerse lo que los define.

Una nueva filosofia de la naturaleza se vislumbra a partir de entender la carne como medio y el espacio ya no como un contenedor sino como construido a partir del propio entrelazo. El camino hacia ella es una ontología de las relaciones como opuesta al dualismo moderno, cuyo punto de partida sea la experiencia sensorial abordada como inmanente a la naturaleza que se pretende explicar. Lo que hay, en Merleau-Ponty es una Sensibilidad en sí en la cual nos encontramos inmersos, que nos constituye: es el entramado común del que estamos hechos nosotros y el mundo

Por lo tanto, el objeto principal de esta nueva filosofia de la naturaleza es devolverle la profundidad y multiplicidad de significados a la noción en

\footnotetext{
${ }^{44}$ Sobre la filosofia de la naturaleza de Whitehead ver: Whitehead, A. N., El concepto de naturaleza, Buenos Aires: Cactus, 2019. Para un estudio crítico sobre el tema: Stengers, I., Pensar con Whitehead: una creación de conceptos libre y salvaje, Buenos Aires: Cactus, 2019.

${ }^{45}$ Merleau-Ponty, M., La nature. Notes et cours du Collège de France, p. 116.

${ }^{46}$ Cf. ibid., p. 125.

${ }^{47}$ Merleau-Ponty, M., Lo visible y lo invisible, p. 125.
} 
cuestión, volviendo a posicionarla en relación a la dimensión experiencial de la cual fue separada. Esto resolvería la reducción mencionada, aquella que por un lado reemplaza la naturaleza por una idea de ambiente constreñido a una ética de los comportamientos y, por el otro, delega exclusivamente la dilucidación de la naturaleza a las ciencias naturales. En tanto carne, el dualismo experiencia-naturaleza escapa a la oposición irreconciliable disolviendo sus límites tajantes y estableciendo fronteras con un cierto espesor. La percepción es considerada un medio que nos hace parte de la naturaleza y fundamenta nuestra relación con ella.

Ya sea del lado de la tradición positivista desvinculando al sujeto humano de ella y transformándola en una pura exterioridad objetiva, o del lado de la ética ambientalista que sólo lo vincula atendiendo a su dimensión comportamental, nos encontramos con un sujeto que experiencia un mundo del cual está desconectado, experiencia una exterioridad. La naturaleza entendida como medio en el mismo sentido de la carne, ayudaría a resignificar la categoría de ambiente a la luz de un pensamiento de la medialidad.

La carne nos abre a un concepto de naturaleza que se extraiga de la propia experiencia, entendida esta última no como la decodificación categorial del dato de los sentidos, sino como el vínculo primordial y punto de partida de toda reflexión. Este, según creemos, es el principal legado para una nueva filosofia de la naturaleza en el marco del materialismo posthumano actual ${ }^{48}$. La noción merleau-pontiana de naturaleza, al compartir el estatuto ontológico de la carne, no se presenta como algo externo al sujeto, sino que es una totalidad abarcativa que borra los limites entre el adentro y el afuera del cuerpo. Esta naturaleza integrada a una teoría de la medialidad construye su significado a partir de las relaciones entre el viviente y el entorno que lo rodea y lo conforma.

\section{Consideraciones finales}

En base a lo explicitado, podemos detectar tres momentos en el desarrollo de la filosofia merleau-pontiana de la medialidad. Primero, un concepto temprano de medio, explicitado principalmente en Fenomenología de la percepción. Segundo, la noción de carne, que es la forma que adopta aquél en

\footnotetext{
${ }^{48}$ Esta idea forma parte de un proyecto mayor, por lo cual, por cuestiones de espacio, nos limitaremos en estas lineas a establecer las bases del problema. En un trabajo inmediatamente posterior, estos planteos serán proyectados a algunos pensadores de la actualidad para analizar esta herencia en el materialismo contemporáneo.
} 
sus desarrollos tardíos. Tercero, el intento de construir un nuevo concepto de naturaleza, interrumpido por la muerte del autor.

Entre los dos primeros momentos hay una diferencia temporal y sus respectivas notas distintivas responden al contexto en que sus planteos fueron explicitados. De este modo, entre una y otra vemos una clara exacerbación del matiz ontológico. A pesar de que, como especificamos en el primer apartado, la Fenomenología se inscribe también dentro de un pensamiento del entre, en Lo visible y lo invisible se afianza un concepto de medio como entrelazo, y no como entorno o medio circundante. El tercer momento también pertenece al pensamiento tardío de Merleau-Ponty, y se encuentra muy cerca de la noción de carne. Para los fines de nuestra investigación, este último modo de entender el concepto nos provee las herramientas para entender la deriva ética de la filosofia de la medialidad.

Además de esto, leer la filosofia de Merleau-Ponty en clave medial suscita no sólo importantes consecuencias ontológicas y éticas sino, como se especificó en el primer apartado, se trazan nuevas directivas epistemológicas y metodológicas. Éstas derivan de los propios compromisos generados por la adopción de una filosofia de la relación. El particular enfoque a partir del cual se abordan los dualismos y la confianza en el encuentro con lo dado a la percepción perfilan de entrada una crítica a la tradición filosófica moderna y sus herencias. En primer lugar, la dualidad es convertida en lo primero, un punto de partida, no un error a resolver. Si bien los componentes son independientes, son mutuamente independientes: lo que resguarda esa independencia es justamente la dependencia de la relación. Lo que los define es esa pertenencia. Segundo, cuando esto es proyectado a la percepción, se evidencia la potencia de esta última como fundamento de toda reflexión filosófica. Pero para que este fundamento sea tal, es necesario rehuir a la pregunta por la cuestión de la veracidad de la experiencia dada a los sentidos.

Cuando el pensamiento se ha posicionado desde afuera de lo sensible, lo ha hecho artificialmente, considerándolo un hecho más del mundo y negando la experiencia propia de la inmersión. Por el contrario, el pensamiento quiasmático requiere de la aceptación de una visión en perspectiva, desde adentro del ser. Como dice Mario Teodoro Ramírez: "Una filosofia carnal, háptica más que óptica, cuestiona totalmente la concepción del vidente como espíritu panorámico o subjetividad de sobrevuelo, y se compromete con una descripción más 
rigurosa y profunda del acto de ver"49. Este compromiso implica no distinguir qué es del mundo y qué es de nosotros. O mejor, nos obliga a reconsiderar ese dualismo (y todo dualismo) como una tensión reciprocamente constitutiva cuyos componentes no se rechazan ni pueden fusionarse, tampoco pueden pensarse por separado sin caer en contradicciones.

En cuanto al núcleo conceptual duro de nuestra investigación, podríamos decir que el recorrido realizado por "El entrelazo - el quiasmo" intentó desarrollar analíticamente el modo en que la noción merleau-pontiana de carne puede ser comprendida desde una filosofia de la relación. La carne, con los tres matices detallados en esta sección, no implica un simple cruce, sino una relación de copertenencia. Hay una continuidad entre mi cuerpo (mi carne) y lo visible (la carne del mundo) y, a la vez, un espacio que media entre cuerpo y mundo, un punto de contacto en constante tensión y armonía. La carne es comprendida como Visibilidad en sí, un modo de ser general que sostiene la tensión antes explicitada. La carne no es lo medial en tanto puente, mera unión, contenedor o transparencia. Es el elemento que da consistencia a todo lo que hay.

Como último paso, la noción de naturaleza sirve de ejemplo para entender el modo en que la teoría merleau-pontiana explicitada nutre con mayor o menor intensidad un gran número de desarrollos actuales en donde opera el pensamiento de lo medial. Es posible concluir entonces, que lo planteado por Merleau-Ponty en torno al quiasmo y la carne es un modo de concebir un ser puro de relación, un tejido que conecta cuerpos y cosas. En efecto, la carne es un medio o médium, lo que esta entremedio y alrededor de las cosas, es la atmósfera que las mantiene unidas y separadas. Es, al igual que lo es el agua o el aire, el elemento en el cual estamos inmersos, que nos mantiene unidos y separados, que nos constituye a nosotros y al mundo.

Recibido: 08/10/2020

Aceptado: 12/02/2021

\footnotetext{
49 Ramírez, M. T., La filosofía del quiasmo. Introducción al pensamiento de Maurice Merleau Ponty, p. 107.
} 


\section{Bibliografia}

Agamben, G., Medios sin fin, Valencia: Pre-textos, 2001.

Agamben, G., Karman. Breve tratado sobre la acción, la culpa y el gesto, Buenos Aires: Adriana Hidalgo Editora, 2018.

Alloa, E., La resistencia de lo sensible, Buenos Aires: Nueva Visión, 2009.

Alloa, E., "The Diacritical Nature of Meaning: Merleau-Ponty with Saussure", en: Chiasmi International, 15 (2013), pp. 167-181. https://doi.org/ 10.5840/chiasmi20131516

Barad, K., Meeting the Universe Halfway: Quantum Physics and the Entanglement of Matter and Meaning, Durham y Londres: Duke University Press, 2007.

Benjamin, W., "La tarea del traductor", en: Angelus Novus, Barcelona: Edhasa, 1971.

Barbaras, R., The Being of the Phenomenon. Merleau-Ponty's Ontology, Indiana: Indiana University Press, 2004.

Braidotti, R., Lo posthumano, Barcelona: Gedisa, 2013.

Carbone, M., The Flesh of Images. Merleau-Ponty between Painting and Cinema, Nueva York: New York University Press, 2015.

Carbone, M., "La naturaleza en Merleau-Ponty: variaciones sobre el tema", en: Devenires, v. I, 2 (2000), pp. 166-185.

Coccia, E., La vida de las plantas, Buenos Aires: Miño y Dávila, 2017.

Combes, M., Simondon. Una filosofia de lo transindividual, Buenos Aires: Cactus, 2017.

Deleuze, G. y F. Guattari, Mil mesetas, Valencia: Pre-textos, 2004.

Deleuze, G., Francis Bacon. Lógica de la sensación, Madrid: Arena Libros, 2016. https:// doi.org/10.30965/9783846760079

Evans, F. y L. Lawlor, Chiasms: Merleau-Ponty's Notion of Flesh, Nueva York: State University of New York Press, 2000.

García, E., Maurice Merleau-Ponty. Filosofia, corporalidad y percepción, Buenos Aires: Rhesis, 2012.

García, E., "¿Qué es un hábito? Acerca de la posibilidad de una desambiguación de la noción merleau-pontiana", en: Ideas. Revista de Filosofía Moderna y Contemporánea, v. III, 6 (2017), pp. 40-71.

Hamrick, W.S. y J. Van der Veken, Nature and Logos. A Whiteheadian Key to MerleauPonty's Fundamental Thought, Nueva York: New York University Press, 2011.

Hass, L., Merleau-Ponty's Philosophy. Indiana: Indiana University Press, 2008.

Merleau-Ponty, M., L'oeil et l'esprit, París: Gallimard, 1964.

Merleau-Ponty, M., Fenomenología de la percepción, Madrid: Planeta, 1993.

Merleau-Ponty, M., La nature. Notes et cours du Collège de France, París: Éditions du Seuil, 1995.

Merleau-Ponty, M., Lo visible y lo invisible, Buenos Aires: Nueva visión, 2010.

Ramírez, M.T., La filosofia del quiasmo. Introducción al pensamiento de Maurice MerleauPonty, Ciudad de México: Fondo de Cultura Económica, 2013.

Toadvine, T., Merleau-Ponty's Philosophy of Nature, Evanston- Illinois: Northwestern University Press, 2009. https://doi.org/10.2307/j.ctv43vs1w

Toadvine, T., "The Chiasm", en: Luft, S. y S. Overgaard (eds.), The Routledge Companion to Phenomenology, Nueva York: Routledge, 2012, pp. 336-347. 